Journal of Computer Science 4 (9): 706-712, 2008

ISSN 1549-3636

(C) 2008 Science Publications

\title{
Object Oriented and Multi-Scale Image Analysis: Strengths, Weaknesses, Opportunities and Threats-A Review
}

\author{
${ }^{1}$ B. Naga Jyothi, ${ }^{2}$ G.R. Babu and ${ }^{3}$ I.V. Murali Krishna \\ ${ }^{1}$ Department of Electronics and Communication Engineering, DMS SVH College of Engineering, \\ Machilipatnam, A.P., India-521002 \\ ${ }^{2}$ Department of Electronics and Communication Engineering, Swami Vivekananda Institute of \\ Engineering andTechnology, Secunderabad, India \\ ${ }^{3}$ Director IST/R and D Center, Jawaharlal Nehru Technological University, Hyderabad, India
}

\begin{abstract}
Problem Statement: In many cases it would be helpful to include already existing data with the GIS for interpretation of remote sensing data. Object Based Image Analysis (OBIA) is a technology for image analysis which addresses exactly those problems hampering a successful bridging of Remote Sensing and GIS. In order to provide potential strategies to meet the proposed objective, a tentative SWOT Analysis is undertaken to identify current Strengths, Weakness, Opportunities and Threats that OBIA faces. Approach: Two different areas had been chosen for testing. The results of pixel based and polygon-based classification has been compared. Results: The classified image derived from polygon based classification is closer to human visual Interpretation. Finally, it is understood that using the e-cognition technique, the weaknesses and threats that OBIA originally suffered are minimized to a great extent. Conclusions: Pixel based classification uses the pure spectral information only. Beyond the pure spectral information, OBIA uses local context information also. This often essential information can be used together with form and texture features of image objects to improve classification significantly.
\end{abstract}

Key words: Object based image analysis, hierarchical network of image objects, semantic networks, image segmentation

\section{INTRODUCTION}

What is OBIA?: In the absence of a formal definition, Object-Based Image Analysis (OBIA) is proposed as a sub-discipline of GIScience devoted to partitioning Remote Sensing (RS) imagery into meaningful imageobjects and assessing their characteristics through spatial, spectral and temporal scale. At its most fundamental level, OBIA requires image segmentation, attribution, classification and the ability to query and link individual objects (A.K.A. segments) in space and time. In order to achieve this, OBIA incorporates knowledge from a vast array of disciplines involved in the generation and use of Geographic Information (GI). It is this unique focus on RS and GI that distinguishes OBIA from related disciplines such as computer vision and biomedical imaging, where outstanding research exists that may significantly contribute to OBIA.

Why is OBIA?: OBIA exists in response to a series of drivers that have transpired over the last decade ${ }^{[2]}$.
- A dramatic increase in commercially available high resolution remote sensing

- imagery $<5.0 \mathrm{~m}$ and the need to develop new markets from these multi-billion dollar investments

- An ever-growing sophistication of user needs and expectations regarding GI products

- Recognition of limitations with pixel-based image approaches (i.e., that pixels are not true geographical objects, that pixel topology is limited, that current remote sensing image analysis largely neglects the spatial photo interpretive elements (i.e., texture, context, shape) and that increased variability implicit within $\mathrm{H}$-Res imagery confuses traditional pixel-based classifiers resulting in lower classification accuracies)

- Increasingly affordable, available and powerful computing tools and a maturing of object-oriented programming

- Increasing awareness that object-based methods can make better use of neglected spatial 
information implicit within RS images and provide greater integration with vector based GIS

- Recognition of the need for multiscale approaches in the monitoring, modelling and management of our environment, for which object-based methods are especially suited. In addition, object-based methods represent viable solutions to the Modifiable Areal Unit Problem (MAUP), of which remote sensing data are a special case

\section{MATERIALS AND METHODS}

Based upon these the suggested conditions are:

- A key objective of OBIA is to develop appropriate theory, methods and tools sufficient to replicate (and or exceed experienced) human interpretation of RS images in automated/semi-automated ways, that will result in increased repeatability and production, while reducing subjectivity, labour and time costs

OBIA uses the fact that important semantic information necessary to interpret an image is mostly not represented in single pixels but in meaningful image objects and their mutual relations.

Delphi 2 creative technologies has developed a new technology and software for object-oriented and multi-scale image analysis. The procedure is based on the so called Fractal Net Evolution approach which is a efficient method.

To describe complex semantics within largely self constructing and dynamic networks. It combines insights in the fractal structure of the world and of semantics with object orientation.

The procedure first extracts image objects which afterwards are classified by means of fuzzy-logic. Basic strategy is to build up a hierarchical network of image objects which allows to represent the image information in different resolutions simultaneously. By operating on the relations between networked objects it is possible to classify local context information. Beyond the pure spectral information this often essential information can be used together with form and texture features of image objects to improve classification significantly.

A basic part is a new patented technique for object segmentation. It extracts image object-primitives in arbitrary resolution-fine or coarse structures-and high quality. This technique has especially been adapted to find image objects in textured data, such as SAR images, high-resolution satellite imagery, airborne data or medical images. Additionally it is used to construct a hierarchical network of image objects, where each object 'knows' its context, its neighborhood and its subobjects.

The knowledge base for analysis comes in form of a hierarchical network of classes. It is threefold and distinguishes between inheritance of features, semantic grouping of classes and collection of classes into structure groups for the purpose of knowledge based object extraction. Features are evaluated by means of next-neighbour and membership functions.

The procedure allows the description and analysis of complex semantic tasks even on textured data. It works on an arbitrary number of channels and allows the treatment of arbitrary data types simultaneously, e.g., different resolution, GIS-layer, alivation. It includes a new method for object oriented texture analysis and object oriented form analysis, both based on the analysis of subobjects. Finally it can be used to extract and export image objects for GIS applications in vectorized form. For different fields of applications, especially bridging remote sensing and GIS, the method proofed its far-reaching potential.

The necessity for integration of remote sensing and GIS: Nowadays GIS is becoming the basic tools to prepare spatially related decisions. But for a GIS database the same fact is true as for each kind of database: It can only be as good and as useful as the quality and actuality of it's data allow. For this reason it would be desirable to utilize results of exploited remote sensing data to a great extend for updating a GIS. The necessity for integration of remote sensing and GIS therefore is always emphasized again.

Especially it would be desirable to do this in a resource and therefore cost-saving way. But unfortunately there are some methodic obstacles preventing extensive automatic processing:

- One is the question of how to gain geo-information directly from remote sensing data. The operationalization of remote sensing is of course in general an objective of many studies and research. But there are two main issues: what to do about the automatic analysis of textured image data such as VHR-data or radar imagery? And, what to do about more complex problems which additionally require the consideration of local context-information?

- The updating of GIS demands polygons of classified image information. But automatic image object extraction is a open field, still today the digitalization of polygons requires in most cases extensive manual resources 
- In many cases it would be helpful to include already existing data with the GIS-for instance cadastral or landscape model data-for interpretation of remote sensing data. But there are no systematic methods which allow image fusion or data fusion for different applications in an operational way

In fact, the described problems are so difficult that up until now one could not speak of an effective integration of remote sensing and GIS.

Object-oriented and multi-scale image analysis in semantic networks: The procedure is based on the so called 'Fractal Net Evolution' approach developed at Delphi2 Creative Technologies which is an efficient method to describe complex semantics within largely self constructing and dynamic networks. For image analysis there are three main points resulting:

- Object orientation: The procedure first extracts image objects which afterwards are classified by means of fuzzy-logic

- Representation of the image information in different scales simultaneously: Each image contains of different semantic levels in the same time. Basic strategy is therefore to build up a hierarchical network of image objects which allows to represent the image information content at different resolutions (scales) simultaneously.

By operating on the relations between networked objects, it is possible to classify local object context information. Beyond the pure spectral information this often essential context information can be used together with form and texture features of image objects to improve classification

- Description, processing and analysis of image information by means of semantic networks

The procedure contains two basic domains:

Construction of a hierarchical network of image objects:

- New procedure for automatic extraction of object primitives in any chosen resolution

- Knowledge based collection of image objects on the basis of classified object primitives

Classification of image objects by means of fuzzy logic:

- On features of objects

- On relations between networked objects operating on the semantic network
Hierarchical network of image objects: A basic part of the procedure is a new technique for object segmentation which is able to find image objects in any chosen resolution (fine or coarse structures) and high quality. This technique has especially been adapted to extract image objects from textured or low-contrast data, such as airborne and satellite SAR and high resolution optical image data, or from medical images. Beyond the pure spectral information, image-objects are characterized by a number of additional features such as texture and form (Fig. 1). All this additional information can hardly be exploited using pixel-based approaches.

The possibility to extract image objects in any chosen scale is an important feature in practical operation: The image object resolution can be adapted to the specific imagery and to the specific problem at hand.

By means of this segmentation technique a hierarchical network of image objects can be constructed in which high resolution objects are subobjects of coarser structures the hierarchical structure represents the information of the image data in different resolutions simultaneously. Each object 'knows' its context, its neighborhood and its subobjects.

Thus, it is possible to define relations between objects, e.g., relative border length to class forest and to utilize this additional and often essential context information.

On the basis of the analysis of subobjects the procedure provides new interesting methods for object oriented texture analysis and object oriented form analysis.

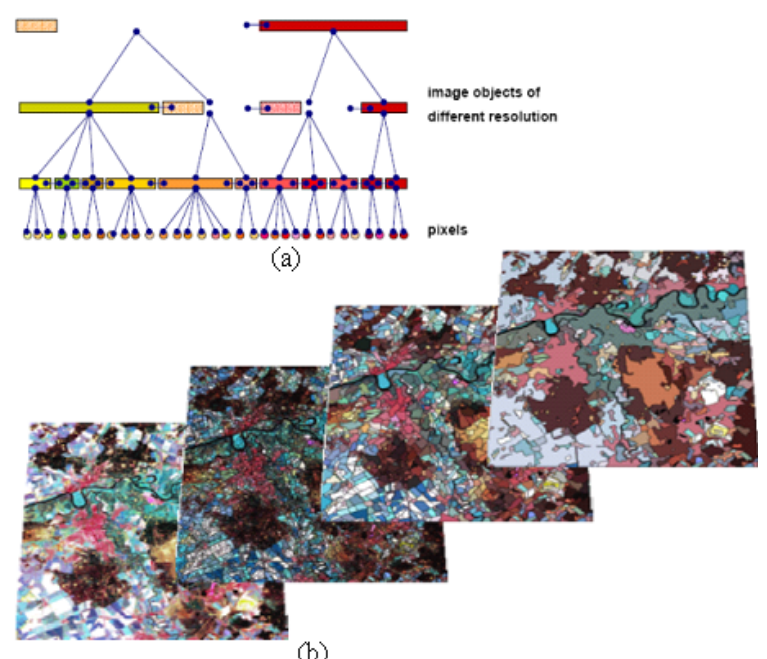

Fig. 1: Representation of image information by means of a hierarchical network of image objects. (a): Schematic view and (b): Image objects on different levels of the hierarchical network 
Classification of image objects: The knowledge basis for analysis comes in the form of a second semantic network, the class hierarchy. It is threefold and distinguishes between inheritance of feature descriptions to subordinate classes, semantic grouping of classes and the collection of classes into structure groups for the purpose of knowledge based object extraction. The advantages are structured Semantics, the possibility to formulate even complex semantics and the possibility for reduction of complexity at formulating a feature description.

Classification is done based on fuzzy logic. The advantage of fuzzy-logic is the possibility to integrate most different kinds of features and to connect them by means of fuzzy logical operators. Thus complex class descriptions are possible. Compared to neural Networks the advantage is a transparent and adaptable set of classification rules. Each single step of classification can be retraced for each image object in detail. The classificators used for the class descriptions are next neighbor or membership functions (Fig. 2).

All steps of an image analysis can be recorded as a complete procedure. Thus, the whole strategy for solving a particular problem can be applied to other data of the same type.

Furthermore, the object oriented approach allows the use of locally different strategies for image analysis depending on the classification of objects.

\section{Advantages of the procedure:}

- Analysis even on textured or low contrast data, e.g. VHR-satellite imagery, airborne or radar data

- Formulation and analysis of complex semantic tasks

- Transparent and adaptable fuzzy logic classification

- Analysis on an arbitrary number of channels

- Data Fusion: analysis of arbitrary data types simultaneously; e.g. different resolution, GIS-layer, elevation

- Export of vectored image objects as classified polygons for GIS applications

SWOT analysis: In order to provide a better understanding of the current state of OBIA and potential strategies to achieve the stated objective, a SWOT Analysis can be undertaken. SWOT Analysis is (one of many possible strategic planning tools) used to evaluate the Strengths, Weakness, Opportunities and Threats involved in a project, or any other situation requiring a decision. Consequently, the objective is to apply this method of planning early in the life cycle of OBIA with the intent that concepts described here can be used to strengthen and guide this emerging discipline.
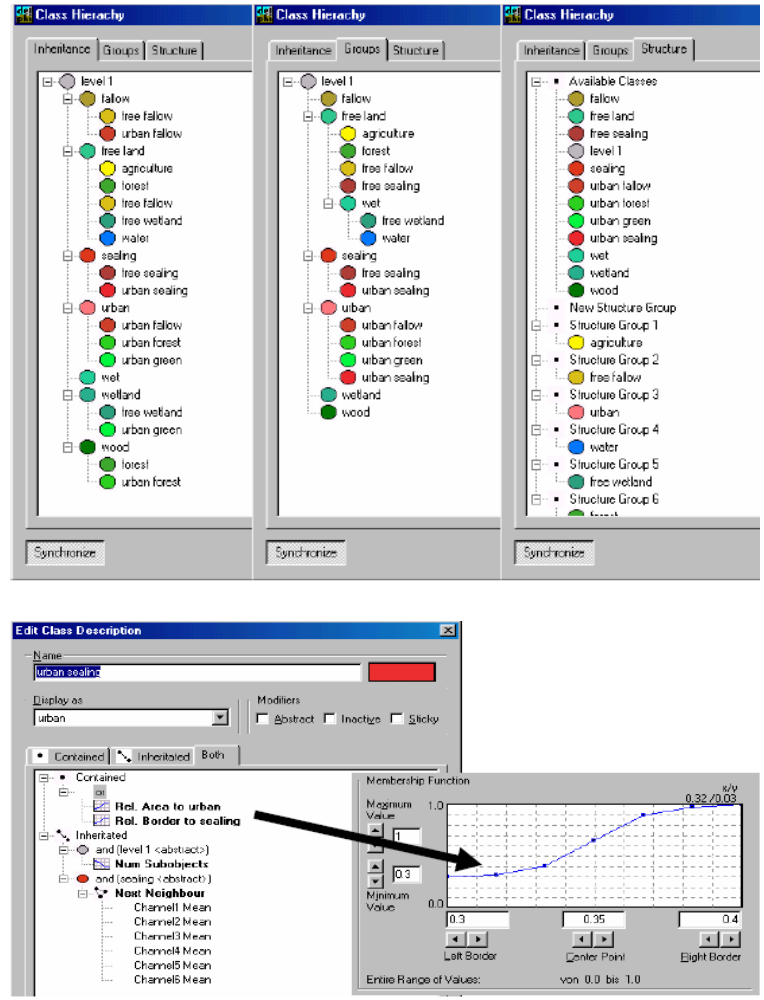

Fig. 2: Class description and membership function

Table 1: SWOT matrix

\begin{tabular}{lll}
\hline & $\begin{array}{l}\text { Helpful to achieving } \\
\text { the objective }\end{array}$ & $\begin{array}{l}\text { Harmful to achieving } \\
\text { the objective }\end{array}$ \\
\hline $\begin{array}{l}\text { Internal (attributes } \\
\text { of the organization) }\end{array}$ & Strength & Weaknesses \\
$\begin{array}{l}\text { External (attributes } \\
\text { of the environment) }\end{array}$ & Opportunities & Threats \\
\hline
\end{tabular}

In practice, once an objective has been established, a multidisciplinary team representing a broad range of perspectives should carry out SWOT analysis; which is typically presented inform of a matrix (Table1).

\section{SWOTs are defined based on the following criteria:}

- Strengths are internal attributes of the organization that are helpful to the achievement of the objective

- Weaknesses are internal attributes of the organization that are harmful to the achievement of the objective

- Opportunities are external conditions that are helpful to the achievement of the objective

- Threats are external conditions that are harmful to the achievement of the objective 
In theory, SWOTs are used as inputs to the creative generation of possible strategies, by asking and answering the following four questions numerous times:

- How can we use each strength?

- How can we stop each weakness?

- How can we exploit each opportunity?

- How can we defend against each threat?

To reap the full benefits of SWOT analysis it is important to use this tool correctly. Firstly, the analysis should provide information that helps in decision making. Consequently, laundry-lists of strengths, weaknesses, are not in themselves very useful. It is only when the potential implications of this information on the organization discipline are assessed that meaningful analysis emerges.

Secondly, it is most beneficial to look at the strengths and weaknesses originating within (i.e., internal to) the discipline or organization. For example, what to do better than anyone else, what where could be improved, what are others likely to see as weakness? Conversely, opportunities and threats should be externally focused i.e., what trends could be taken advantage of, how to turn strengths into opportunities, what trends could do harm ${ }^{[3]}$ ?

\section{OBIA strengths:}

- Partitioning an image into objects is akin to the way humans conceptually organize the landscape to comprehend it

- Using image-objects as basic units reduces computational classifier load by orders of magnitude, and at the same time enables the user to take advantage of more complex techniques (e.g., non-parametric)

- Image-objects exhibit useful features (e.g., shape, texture, context relations with other objects) that single pixels lack

- Image-objects are less sensitive to MAUP ${ }^{[4]}$ than units that do not keep a correspondence with the structure of the phenomenon under study

- Image-objects can be more readily integrated in vector GIS than pixel-wise classified raster maps

- Several OBIA methods/commercial software packages build upon the powerful Object-Oriented (OO) paradigm

\section{OBIA weaknesses:}

- Under the guise of 'flexibility' current commercial object based software provides overly complicated options
- There are numerous challenges involved in processing very large datasets. Even if OBIA is more efficient than pixel-based approaches, segmenting a multispectral image of several tens of mega-pixels is a formidable task (efficient tiling multiprocessing solutions are necessary)

- Segmentation is an ill-posed problem, in the sense it has no unique solution, e.g., (i) changing the bit depth of ones heterogeneity measure can lead to different segmentations. (ii) Also even human photo-interpreters will not delineate exactly the same things

- There is a lack of consensus and research on the conceptual foundations of this new paradigm, i.e., on the relationship between image-objects (segments) and landscape objects (patches). For example, (i) what is the basis to believe that segmentation-derived objects are fine representations of landscape structural-functional units? (ii) How do one know when the segmentation is good? (iii) Is there a formally stated and accepted conceptual foundation?

- There exists a poor understanding of scale and hierarchical relations among objects derived at different resolutions

\section{OBIA opportunities:}

- Object-Oriented concepts and methods have been successfully applied to many different problems, not only computer languages, and they can be easily adapted to OBIA. This integration not only includes $\mathrm{OO}$ programming, but all the corpus of methods and techniques customarily used in biomedical imaging and computer vision that remain unknown to most of the remote sensing community

- While further research is needed, interesting integrative object-based proposals already exist that offers ontological foundations ${ }^{[1]}$

- There are new IT tools that may accelerate consensus and cohesion of OBIA

- There is a steadily growing community of RS GIS practitioners that currently use image segmentation for different GI applications. Thus, as OBIA matures, new commercial research opportunities will exist to tailor object-based solutions for specific fields, disciplines and user needs i.e., forestry, habitat mapping, urban mapping, mineral exploration, transportation, security.

- Symmetric multiprocessing, parallel processing and grid computing are recent technologies that OBIA methods may build upon to tackle problems related to the analysis of large datasets 


\section{OBIA threats:}

- OBIA is far from been an operationally established paradigm, yet many users of commercial OBIA software do not recognize this fundamental fact. OBIA is not one specific research or commercial software. Much remains to be solved

- Trying to make distinct OBIA from other OO concepts and methods (e.g., by using 'based' instead of 'oriented') may contribute to insulation (of users in an esoteric world of objects) and isolation (of the concept) rather than to consolidation

- The visual appeal of image-objects, their easy GISintegration and the enhanced classification possibilities have attracted the attention of major RS image processing vendors, who are increasingly incorporating new segmentation tools into their packages. This provides a wider choice for practitioners, but promotes confusion (among different packages, options and syntax) and makes it more difficult to reach a consensus on what OBIA is all about. Will a lack of protocols, formats, and standards lead to a segmentation of the field rather than a consolidation?

\section{RESULTS}

In this study, two different areas have been chosen for testing the new technique. The first one is located at urbanization area as seen in part (a), Fig. 4. The other one is located at high land, vegetation area, which surrounded by dense typical forest as seen in part (b), Fig. 4. The results of pixel based and polygon-based classification has been compared. It is shown in part (c), (d), (e) and (f), Fig. 4. Figure 3 shows hierarchical net of image objects derived from image segmentation.

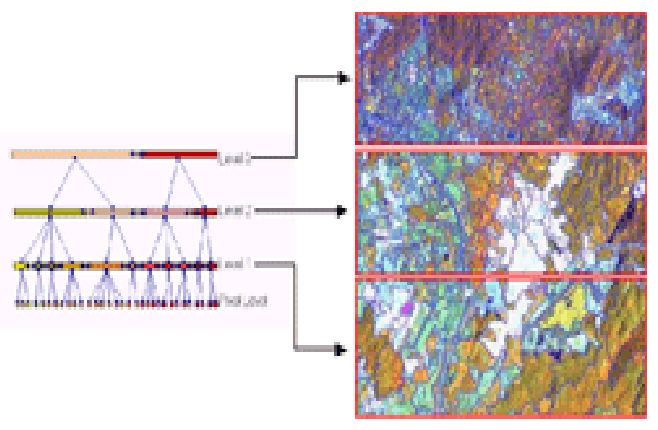

Fig. 3: Hierarchical net of image objects derived from image segmentation level 1 (5 pixels scale parameter), level 2 (15 pixels scale parameter) and level 3 (30 pixels scale parameter)

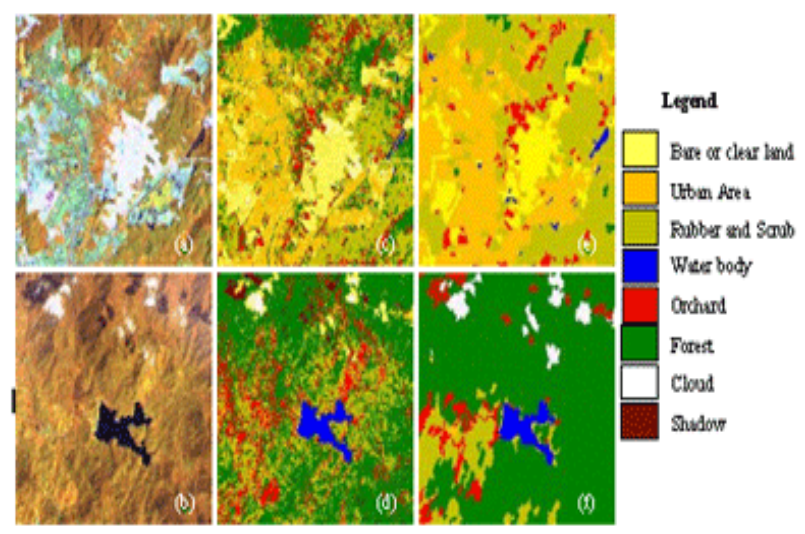

Fig. 4: Landsat TM (Band 4, 5, 3) testing area (a) Urbanization Area; (b) High Land Vegetation Area. Comparison between pixel based and polygon based classification (c) pixel-based at urbanization area; (d) pixel-based at vegetation area; (e) polygon-based at urbanization area; (f) polygon-based at vegetation area

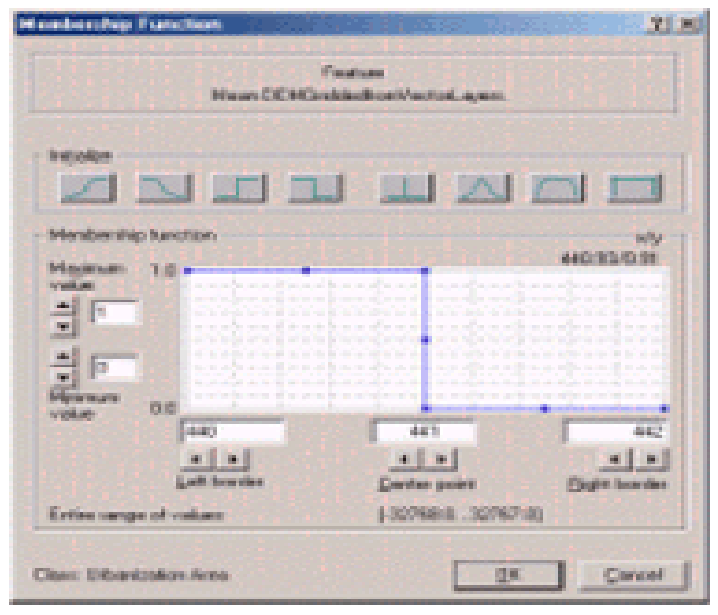

Fig. 5: Setting the DEM parameter to determine the urbanization area

The parts (c) and (d) from Fig. 4 are the results of pixel-based maximum likelihood supervised classification. As a result, the classified image produced salt and pepper image or lot of small clumps $(<10$ pixels) appeared in the classified image. Vice versa, the classified image derived from polygon-based classification is closer to human visual interpretation. The pixel-based classification results, which only based on the spectral mean of the digital number itself, is no way to differentiate cloud with urban and clear land completely [see Fig. 4, part (d)]. But if looked carefully 
on high land vegetation area at Fig. 4, part (f), it can be clearly seen that cloud is no more mix classified, neither with urban area nor bare or clear land. In e-cognition, the classification is not only based on the spectral number itself, it can accept other source regardless of it's data properties ( 8 bits, 16 bits or 32 bits). In this case, the cloud is clearly classified by using the DEM band (16 bits) as the main parameter where in generally no urban area or clear land can be found at certain height especially at hilly area where its' surrounding area cover by dense typical forest.

Due to this, by setting the parameter at the urbanization area can only be found lower than $440 \mathrm{~m}$ from the sea level only as shown in Fig. 5.

\section{DISCUSSION}

In order to make a direct comparison of accuracy assessment between the pixel-based and polygon-based classification results, the accuracy assessment has been carried out in the same environment. The program automatically picks out 300 random sample points plus 15 ground truth points for accuracy assessment. The statistic result is shown in Table 2. From the results, the overall accuracy has shown the higher accuracy in polygon-based classification result.

Table 2: Accuracy assessment

\begin{tabular}{llr}
\hline Accuracy & Statistics pixel-based (\%) & Polygon-based (\%) \\
\hline Forest & 81.507 & 94.972 \\
Water body & 94.118 & 100.000 \\
Urban area & 88.889 & 86.111 \\
Bare or clear land & 72.727 & 83.333 \\
Orchard & 75.000 & 84.000 \\
Rubber and scrub & 84.906 & 82.353 \\
Cloud & 83.333 & 100.000 \\
Shadow & 33.333 & 100.000 \\
Overall accuracy & 81.667 & 90.667 \\
\hline
\end{tabular}

\section{CONCLUSION}

In this study, the object oriented analysis technique has been introduced for classification and the result is obtained for land cover mapping. The proposed technique using e-cognition was successfully tested with Landsat TM image. The results presented in this study show the efficiency and higher accuracy for polygon-based classification thus over coming the weaknesses OBIA was found to have originally. This technique is recommended to test on VHR data such as Ikonos image or Aerial photos especially in town area where more details classes can be generated.

Efforts are made to provide an early attempt at formally defining Object-Based Image Analysis
(OBIA), outlining a key objective of this new field, and identifying a number of Strengths, Weakness,

Opportunities and Threats (SWOT) that OBIA faces, with the intent that items described here can be used to strengthen and guide this promising new field. A key issue faced by OBIA is to ensure that an integrative, well understood and easily defined ontology is developed and integrated within the research and commercial software that is being developed for object-based image analysis.

As a sub-discipline of GIScience, this ontology can draw upon the strong theoretical and application based components that already exist in the established fields of remote sensing, image processing, computer vision, computer science, and landscape ecology.

The Fractal Net Evolution procedure described above for semantic networks solves some main problems encountered to date whenever geoinformation is to be extracted from remote sensing imagery applying standard, pixel-based operations. This especially includes main problems of the integration of remote sensing and GIS.

\section{ACKNOWLEDGEMENT}

The ${ }^{1}$ author expresses humble and sincere thanks to the supervisors for their constant guidance and support during the preparation of this presentation.

\section{REFERENCES}

1. Hay, G.J., G. Castilla, M. Wulder, M.A. and J.R. Ruiz, 2005. An automated object-based approach for the multiscale image segmentation of forest scenes. Int. J. Applied Earth Observ. Geoinform., 7: 339-359. DOI: 10.1016/j.jag. 2005.06.005.

2. Benz, U.C., 1999. Supervised fuzzy analysis of single and multi-channel SAR data. IEEE TGARS., 37: 1023-1037. DOI: 10.1109/36.752221.

3. Cloude, S.R. E. Pottier, 1996. A review of target decomposition theorems in radar polarimetry. IEEE TGRS, 34: 498-518. DOI: 10.1109/36.485127.

4. Dong, Y., A.K. Milne, B.C. Forster, 2001. Segmentation and classification of vegetated areas using polarimetric SAR image data. IEEE TGARS, 39: 321-329. DOI: 10.1109/36.905240.

5. Lee, J. S. M.R. Grunes, G. de Grandi, 1999. Polarimetric SAR speckle filtering and its implication for classification. TGARS, 37: 23632373. DOI: $10.1109 / 36.789635$. 